\title{
Large number of receptors may reduce cellular response time variation
}

\author{
Xiang Cheng, Lina Merchan, Martin Tchernookov \\ Department of Physics, Emory University, Atlanta, GA 30322, USA
}

\begin{abstract}
Ilya Nemenman
Departments of Physics and Biology, Computational and Life Sciences Initiative, Emory University, Atlanta, GA 30322, USA

E-mail: xiang.cheng@emory.edu,ilya.nemenman@emory.edu
\end{abstract}

\begin{abstract}
Cells often have tens of thousands of receptors, even though only a few activated receptors can trigger full cellular responses. Reasons for the overabundance of receptors remain unclear. We suggest that, in certain conditions, the large number of receptors results in a competition among receptors to be the first to activate the cell. The competition decreases the variability of the time to cellular activation, and hence results in a more synchronous activation of cells. We argue that, in simple models, this variability reduction does not necessarily interfere with the receptor specificity to ligands achieved by the kinetic proofreading mechanism. Thus cells can be activated accurately in time and specifically to certain signals. We predict the minimum number of receptors needed to reduce the coefficient of variation for the time to activation following binding of a specific ligand. Further, we predict the maximum number of receptors so that the kinetic proofreading mechanism still can improve the specificity of the activation. These predictions fall in line with experimentally reported receptor numbers for multiple systems.
\end{abstract}

PACS numbers: 87.16.Xa, 87.17.Aa, 87.15.R

Keywords: first passage time, cooperativity, noise suppression, kinetic proofreading, specificity, spare receptors

\section{Introduction}

Cellular responses to changes in the surrounding world are mediated by protein signaling pathways. These pathways are typically activated by modification of cell surface receptors. For example, receptors on the surface of immune cells are a key component in detecting pathogens and activating cellular proliferation, degranulation, and other immune responses. The number of such receptors on the cell surface typically is $10^{4} \ldots 10^{6}[1,2$, 3] , Surprisingly, sometimes as few as a hundred or even fewer bound

$\ddagger$ These and subsequent numbers have to be taken with a grain of salt since, first of all, they represent a compilation of different systems in different organisms and cell lines. Secondly, a variety of processes 
receptors are needed to fully activate a cell. For example, T-cells can be activated by 1. . 10 receptors at the T-cell-B-cell synapse [10, 11]. Rat basophilic leukemia cells require fewer than $5 \%$ active Fce-RI receptors to degranulate [12]. This is not unique to immune receptors. For example, cellular response can be triggered by only a few active estrogen receptors [13]. This phenomenon of cells having an excessive number of receptors compared to what is needed for activation has been known for a while as a problem of "spare receptors" [14, 15]

Functional importance of this overabundance of receptors is not understood, and several explanations can be considered. One possibility is that the chemical information may not be distributed uniformly in space, requiring receptors at multiple locations on the cell surface. An example is the T-cell, which is commonly activated by antigenpresenting cells through a localized synapse [16]. Alternatively, the number of ligands may be very low, and then the probability of receptor-ligand binding is increased by having many receptors even in the well-mixed chemical kinetics limit [10]. Finally, large number of receptors facilitates clustering, promoting collective response, which may lead to signal amplification [17, 18].

Here we suggest that, in an alternative regime of a high ligand concentration, the large number of receptors may play an additional role. For example, for biological processes such as the immune response, synchronous and short cellular activation times would allow a concerted defense against an infection, and may be functionally advantageous. Similarly, concerted response is important in growth receptor signaling. We suggest that such improvement in the accuracy of the activation time may be achieved through a competition among many receptors.

We envision a receptor that, when activated, produces an active messenger molecule. A few, and maybe just one, of such molecules are sufficient to start transcription or otherwise activate the whole cell. Each receptor takes an extended and variable amount of time to activate. Then the first few receptors out of many that have achieved full activation will activate the cell. The variability of the activation time of these fastest receptors can be smaller than that of a typical receptor. To build an intuition, think about the distribution of finish times for runners in a marathon. In this analogy, each cell is a marathon with many receptors representing runners. They all start at about the same time, launched into the activation race by an abundant ligand, and activated receptors are represented by runners who have finished the race. In large marathons with thousands of participants, the time it takes to reach the finish line for the first few runners is much smaller than the mean of the finishing times. More importantly, for an individual marathon, the variability in finish times among all the

complicate the picture. For example, prior ligand exposure history may influence the number of receptors and of associated kinases, hence controlling the system's gain [4, 5. Further, receptors may form aggregates that activate collectively, so that the ability of a receptor to activate a cell is a function of its physical context [6, 7, 8, Finally, serial engagement allows multiple receptor (and cell) activations by the same ligand molecule $[9$. Nonetheless, we believe these numbers to be correct within an order of magnitude, and hence illustrative. 
runners can be fairly large, of order of several hours. And yet the distribution of times of the winners of many marathons has the variability of the order of minutes. In a similar manner, the receptors that are activated first in different cells might have activation times with small variance, leading to a more synchronous response.

In this work, we approach the problem in the context of simplified receptor models. The receptor activation is first modeled as a linear chain of $L$ states, such as ligand binding, conformational changes, dimerization, etc. Ligand presentation starts the progression along the chain, and the final state represents receptor activation. With this model, the probability distribution of time to activation of the first receptor out of $N$ receptors, with $N \gg 1$, is given by a Weibull distribution, which has a small coefficient of variation. When $m(1 \ll m \ll N)$ receptors are needed to activate the cell, the coefficient of variation decreases even further. This "racing to activation" results in a short and less variable time to activation.

Finally, we point out that, to activate specifically to a certain ligand, cells often use kinetic proofreading [19, 20, 21]. We show that a large number of receptors does not necessarily interfere with the ability to proofread. It enables cells to be activated temporally precisely, but also specifically to certain ligands. Using biologically reasonable parameter values, we predict the number of receptors that allows for both of these properties.

(a) Multistep Activation

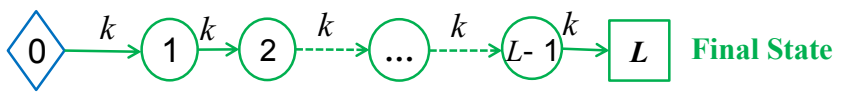

(b)

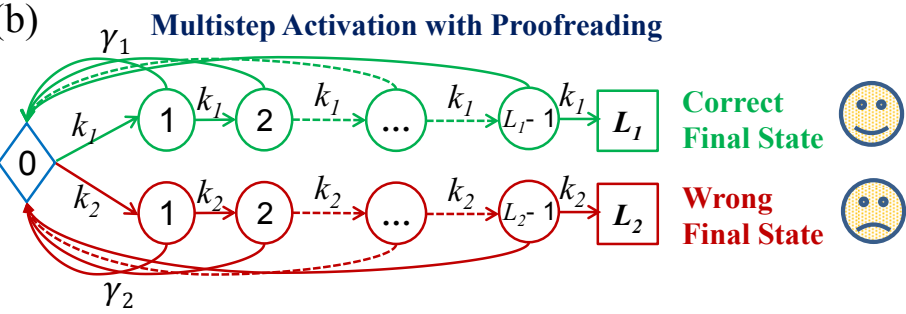

Figure 1. Schematic description of the model. (a) $L$ irreversible steps with rates $k$ are needed to activate a receptor. (b) Kinetic proofreading, denoted by the backwards transitions with the rate $\gamma$, allows the receptor preferentially by a correct ligand.

\section{Multistep Activation}

A simplified model of receptor activation consists of a linear chain of $L$ irreversible events where the receptor undergoes biochemical transformations that ultimately lead to activation, see Fig. 17. In this figure, the multistep activation process begins at the diamond (site $i=0$ ), and at each site the system can transit one step towards activation with a forward rate $k$. Reaching the right-most site, $i=L$, will lead to the receptor activation irreversible on the time scales considered. In order to simplify the algebraic 
expressions, we assume an equal forward rate for all sites. This simplification may reduce the generality of our results somewhat, but it does not change their dependence on $L$, provided none of the rates $k$ are small enough to form a bottleneck.

The probability distribution of the time to complete each single step in the process of the multistep activation is an exponential distribution with rate $k$

$$
p(t)=k \exp (-k t) .
$$

The mean time is $\mu=1 / k$, the variance is $\sigma^{2}=1 / k^{2}$, and the coefficient of variation is c.v. $=1$. For the whole process with $L$ steps, the probability distribution of time to activation is the well known $\Gamma$ distribution

$$
P_{1}(t)=\frac{k^{L}}{(L-1) !} \exp (-k t) t^{L-1}
$$

This distribution has a broad peak. An example of the $\Gamma$-distribution with $L=16$ and $k=1$ is shown in Fig. 2. The moments of the $\Gamma$ distribution are readily available:

$$
\begin{aligned}
\mu & =L / k, \\
\sigma^{2} & =L / k^{2}, \\
\text { c.v. } & =1 / \sqrt{L} .
\end{aligned}
$$

The observation that multistep chemical reactions reduce the coefficient of variation of activation time, has been well understood computationally and experimentally, most notably in the context of vertebrate vision [22].

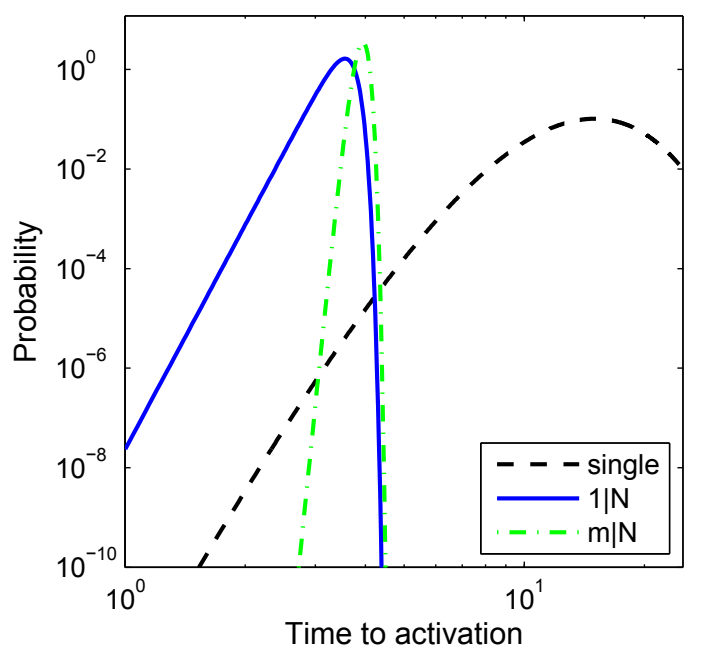

Figure 2. The probability distributions of time to activation with no kinetic proofreading. We use $L=16, k=1, N=30,000$, and $m=5$. The distribution is shown for a single receptor (dashed line), the first receptor out of $N$ (solid), and the first $m$ receptors out of $N$ (dash-dotted).

The decrease of the coefficient of variation in proportion to $1 / \sqrt{L}$ holds true for a single receptor. However, a cell usually has tens of thousands of receptors, $N \gg 1$. We are interested in understanding how this large number affects the mean and the 
variability of the activation times. The activation time distribution for the first receptor out of $N$ receptors is

$$
P_{1 \mid N}(t)=N P_{1}(t)\left(1-\int_{0}^{t} P_{1}\left(t^{\prime}\right) d t^{\prime}\right)^{N-1}
$$

where $P_{1}(t)$ is the $\Gamma$ distribution shown in Eq. (2). Here $P_{1}(t)$ stands for the probability of activating the first receptor at exactly the time $t$, and there are $N$ choices of this receptor. The last term represents that the other $N-1$ receptors must not be activated before $t$. For $N \gg 1, P_{1 \mid N}$ can be simplified since it is almost certain that the first receptor is activated at the left tail of the $\Gamma$ distribution $\left(t \ll \frac{L-1}{k}\right)$, although a typical receptor takes a much longer time to be activated. Thus, the distribution $P_{1 \mid N}$ can be rewritten as

$$
P_{1 \mid N}(t) \approx \frac{L}{\alpha}\left(\frac{t}{\alpha}\right)^{L-1} \exp \left[-\left(\frac{t}{\alpha}\right)^{L}\right] .
$$

where $\alpha=\left(\frac{L !}{N k^{L}}\right)^{\frac{1}{L}} \cdot P_{1 \mid N}$ is the Weibull distribution, which we illustrate in Fig. 2. The Weibull distribution has the following well-known statistical properties

$$
\begin{aligned}
\mu & =\alpha \Gamma\left(1+\frac{1}{L}\right), \\
\sigma^{2} & =\alpha^{2}\left[\Gamma\left(1+\frac{1}{L}\right)-\Gamma^{2}\left(1+\frac{1}{L}\right)\right], \\
\text { c.v. } & =\frac{\sqrt{\psi^{(1)}(1)}}{L} \approx \frac{1.28}{L},
\end{aligned}
$$

where $\Gamma(x)$ is the $\Gamma$ function and $\psi^{(1)}(x)$ is a polygamma function defined as $\psi^{(1)}(x)=$ $\frac{\mathrm{d}^{2}}{\mathrm{~d} x^{2}} \ln \Gamma(x)$. The coefficient of variation goes as $\propto \frac{1}{L}$, which decreases much faster with $L$ than it does for a single receptor activation, $\frac{1}{\sqrt{L}}$.

One can envision a situation where a cooperative action of a few receptors, or more precisely of their activated messages, is needed to activate the whole cell. We can extend our model by studying the distribution of time to activating $m$ out of $N$ receptors, $P_{m \mid N}(t)$, with $1 \ll m \ll N$. There are $\left(\begin{array}{l}N \\ m\end{array}\right)$ possibilities to choose the set of $m$ receptors to be activated out of total $N$ receptors. We require that one of the $m$ receptors activates at exactly time $t$, which has a probability of $m P_{1}(t)$. The other $m-1$ receptors will finish the process at some point before time $t$ which is described by $\left[\int_{0}^{t} P_{1}\left(t^{\prime}\right) d t^{\prime}\right]^{m-1}$. The rest of the $N-m$ receptors are not activated up to time $t$. Combining the terms, we get

$P_{m \mid N}(t)=\frac{N !}{m !(N-m) !} m P_{1}(t)\left[\int_{0}^{t} P_{1}\left(t^{\prime}\right) d t^{\prime}\right]^{m-1}\left[1-\int_{0}^{t} P_{1}\left(t^{\prime}\right) d t^{\prime}\right]^{N-m}$.

With the same approximation $t \ll \frac{L-1}{k}, P_{m \mid N}(t)$ can be rewritten as

$$
P_{m \mid N}(t) \approx \frac{N !}{m !(N-m) !} m P_{1}(t)\left(\frac{k^{L}}{L !}\right)^{m} t^{m L-1} \exp \left(-\frac{N k^{L}}{L !} t^{L}\right) .
$$




\begin{tabular}{|c|c|c|c|c|}
\hline & Mean time & Variance & c.v. & example of c.v. \\
\hline 1 receptor & $\frac{L}{k}$ & $\frac{L}{k^{2}}$ & $\frac{1}{\sqrt{L}}$ & $25.0 \%$ \\
\hline $1 \mid N$ receptors & $\alpha \Gamma\left(1+\frac{1}{L}\right)$ & $\alpha^{2}\left[\Gamma\left(1+\frac{2}{L}\right)-\Gamma^{2}\left(1+\frac{1}{L}\right)\right]$ & $\sim \frac{1.28}{L}$ & $8.0 \%$ \\
\hline$m \mid N$ receptors & $\frac{1}{(m-1) !}\left[\frac{L !}{N}\right] \frac{1}{L} \Gamma\left(m+\frac{1}{L}\right) \frac{1}{k}$ & $\frac{1}{(m-1) !}\left[\frac{L !}{N}\right] \frac{2}{L} \Gamma\left(m+\frac{2}{L}\right) \frac{1}{k^{2}}$ & $\sim \frac{1}{L \sqrt{m}}$ & $2.8 \%$ \\
\hline
\end{tabular}

Table 1. Comparison of activation time distribution for three different activation processes. The example uses the values $L=16, k=1, m=5$, and $N=30,000$

This distribution is shown in Fig. 2 with the usual parameter values, and $m=5$. Activating multiple receptors has a narrower distribution with a smaller coefficient of variation. We calculate

$$
\begin{aligned}
& \mu \approx \frac{1}{(m-1) !}\left(\frac{L !}{N}\right)^{\frac{1}{L}} \Gamma\left(m+\frac{1}{L}\right) \frac{1}{k}, \\
& \sigma^{2} \approx \frac{1}{(m-1) !}\left(\frac{L !}{N}\right)^{\frac{2}{L}} \Gamma\left(m+\frac{2}{L}\right) \frac{1}{k^{2}}, \\
& \text { c.v. } \approx \frac{\sqrt{\psi^{(1)}(m)}}{L} \approx \frac{1}{L \sqrt{m}},
\end{aligned}
$$

where the last approximation comes from the first few terms in the asymptotic expansion of $\psi^{1}(m) \approx 1 / m-1 / 2 m^{2}+1 / 24 m^{3}$, assuming $m \gg 1$. Thus the coefficient of variation for cooperative activation is further reduced compared to the activation by the first receptor.

The results of the three discussed cases (single receptor, the first out of many receptors, and the first $m$ out of many receptors needed for activation) are summarized in Table 1. The racing to activation mechanism with a large number of participating receptors can reduce the coefficient of variation of the activation time to below $5 \%$ for reasonable parameters. This could mean a more synchronous activation of cells.

\section{Multistep activation with kinetic proofreading}

Receptors can bind many non-specific ligands, and they employ kinetic proofreading to increase specificity and activate predominantly following binding of a specific ligand [21, 23]. Our model of kinetic proofreading follows Ref. [24], and is shown in Fig. 11b. We assume that there are two branches of sequential events in the receptor state diagram: a correct branch and a wrong one. The two branches represent activation sequences following binding of a specific/nonspecific ligand molecules, with rates $k_{1}$ and $k_{2}$ for the correct/wrong branches, respectively. For both branches, kinetic proofreading is represented by possible jumps back to the initial state with rates $\gamma_{1}$ and $\gamma_{2}$, respectively. These jumps can correspond to ligand unbinding from the receptor, followed by return of the receptor into the initial, fully inactive state. If $\gamma_{2} \gg \gamma_{1}$ (that is, a nonspecific ligand unbinds faster than the specific one), then the receptor has a much lower probability to reach the final, absorbing state along the wrong branch than along the correct one, 
hence increasing the specificity. While this model is crude, it nonetheless captures the basic physical properties of the kinetic proofreading process.

Kinetic proofreading typically leads to a near-exponential distribution of completion times [24, 25], which has c.v. $\approx 1$. The distribution is different from the exponential only over a very short initial time period since it is difficult to traverse the sequence of $L$ steps to activation quickly, see Fig. 3. This has a potential of interfering with the racing to activation mechanism for improving temporal precision. Indeed, a receptor with an exponentially distributed activation time is equivalent to a single-step receptor, $L=1$. In this case the corresponding $\Gamma$ and the Weibull distributions are exponential as well, providing no reduction in the coefficient of variation.

However, if the number of receptors is sufficiently large, then the first few receptors will have a substantial chance to activate during the initial, nonexponential phase of the completion time distribution. That is, they will activate quickly in a linear sequence of events, as in the previous Section, having no time to revert back to the initial state through the proofreading mechanism. Thus if the number of receptors is sufficient to complete so quickly along the correct branch, the temporal precision will increase through the racing to activation mechanism. On the other hand, since $\gamma_{2}$ is larger, the receptor trying to activate over the same time along the wrong branch may have sufficient time to revert to the initial state repeatedly, falling into the exponential part of the completion time distribution. This suggests that the number of receptors on the cell surface should also be limited from above, so that wrong activation has a low probability of happening in the pre-exponential part of the activation distribution.

Mathematically, we can summarize these arguments as follows. Following Ref. [25], we approximate the probability distribution of completing along a single branch of the state space as:

$$
\begin{array}{ll}
P(t) \approx \frac{k^{L}}{(L-1) !} t^{L-1} \exp [-(\gamma+k) t], & t \ll t_{C}, \\
P(t) \approx \frac{1}{\mu} \exp \left(-\frac{t}{\mu}\right), & t \gg t_{C} .
\end{array}
$$

Here the short-time limit starts as the $\Gamma$-distribution, and there is an additional decrease in probability of completing at time $t$ by $\exp (-\gamma t)$ because of kinetic proofreading. This approximation is valid to the left of its maximum,

$$
t_{C}=\frac{L-1}{k+\gamma}
$$

Further, $\mu$ is the mean activation time, calculated in Ref. [25] to be

$$
\mu=\frac{1}{\gamma}\left[\left(1+\frac{\gamma}{k}\right)^{L}-1\right]
$$

The approximation is illustrated in Fig. 3 .

The variance reduction along the correct branch requires that the first receptors 


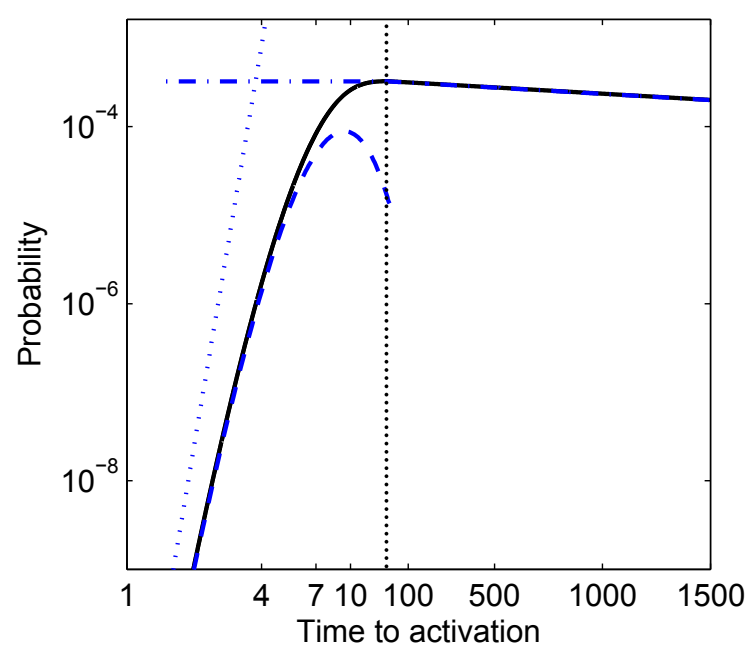

Figure 3. The probability distribution of time to activation with kinetic proofreading for a single branch with $L=16, k=1$, and $\gamma=0.6$. The vertical dotted line is the $t$ of maximum probability. On left side of this line, the curves are in log-log scale; while the curves on the right are in log-linear scale. Solid line comes from a numerical solution of the master equation describing the receptor. We see the power law distribution when $t$ is small, and the exponential distribution when $t$ is large. These are illustrated with the right tail exponential asymptotic, Eq. 17) (dash-dotted line); the left tail asymptotic, Eq. 16 (dashed line), and the power law line $k^{L} t^{L-1} /(L-1)$ ! (dotted line).

are activated before $t_{C_{1}}$, that is:

$$
N \int_{0}^{t_{C_{1}}} \frac{k_{1}^{L_{1}}}{\left(L_{1}-1\right) !} t^{L_{1}-1} \exp \left[-\left(k_{1}+\gamma_{1}\right) t\right] d t \gg 1,
$$

where, as before, the subscript 1 stands for the correct branch. Using the standard expressions for the incomplete $\Gamma$-function, we thus derive the minimum number $N_{\min }$ of receptors needed for variability reduction along the correct branch

$$
N \gg N_{\min }=\frac{\left(1+\frac{\gamma_{1}}{k_{1}}\right)^{L_{1}}}{1-\exp \left(1-L_{1}\right)\left(\sum_{i=0}^{L_{1}-1} \frac{\left(L_{1}-1\right)^{i}}{i !}\right)} .
$$

This sets the lower bound on the number of receptors on the cell surface. However, if the number of receptors was too high, some of the ones bound by incorrect ligands would activate in the left tail of the distribution, before the kinetic proofreading has a chance to kick in. The requirement that the probability of early activation over the wrong branch among all receptors is negligible sets the upper limit on the receptor number:

$$
N \int_{0}^{t_{C_{2}}} \frac{k_{2}^{L_{2}}}{\left(L_{2}-1\right) !} t^{L_{2}-1} \exp \left[-\left(k_{2}+\gamma_{2}\right) t\right] d t \ll 1,
$$


where the subscript 2 stands for the wrong branch. This results in

$$
N \ll N_{\max }=\frac{\left(1+\frac{\gamma_{2}}{k_{2}}\right)^{L_{2}}}{1-\exp \left(1-L_{2}\right)\left(\sum_{i=0}^{L_{2}-1} \frac{\left(L_{2}-1\right)^{i}}{i !}\right)} .
$$

While we do not expect that the bounds derived from our manifestly oversimplified model predict the real number of receptors on the cell surface, it is still worthwhile to verify if the obtained bounds are meaningful, so that kinetic proofreading over a wrong branch can be consistent with narrow activation time over the correct one. As in Ref. [25], we choose $L=16$, and $k_{1}=k_{2}=1$, the same for both the correct and the incorrect branches. To choose the kinetic proofreading rates $\gamma_{1,2}$, we note that they correspond, for example, to unbinding of a ligand from the receptor complex, so that the log-ratio of the rates are related to the difference of the binding free energies $\Delta G_{1,2}$ of the correct and the incorrect ligands:

$$
\Delta G_{1}-\Delta G_{2}=k_{B} T \ln \frac{\gamma_{2}}{\gamma_{1}}
$$

Assuming the difference in the binding free energies of a few thermal energies, we get

$$
\frac{\gamma_{2}}{\gamma_{1}} \sim 2 \ldots 10
$$

Now choosing $\gamma_{1} \approx 0.7$, we get the minimum number of receptors $N_{\min } \sim 10^{4}$, and the maximum number $N_{\max } \sim 10^{7}$ or more. The window between $N_{\min }$ and $N_{\max }$ is sufficiently large to allow the choice of receptor number that satisfies both the proofreading and the narrow activation time. Further, these numbers are realistic in the context of biological receptor numbers, which range between $10^{4}$ and $10^{6}$.

\section{Conclusion}

Biological systems must operate under constraints posed by the physical world. One of such constraints is robustness to intrinsic noise that comes about from the small number of stochastically activated molecular components in cellular networks. Performing averages of different kinds (over time, space, or different molecular species) is, essentially, the only way of ensuring such robustness. In this context, it has been understood for many years that sequential activation of $L$ molecular components averages the times of the activation steps and reduces the coefficient of variation of the time to activation, which scales then as $\sim 1 / \sqrt{L}[26$ ]. This has been observed experimentally in the context of vertebrate phototransduction [22]. Here we propose a mechanism that can further reduce the variability to $\sim 1 / L$, and even $\sim 1 / L \sqrt{m}$ (where $m$ is the cooperativity of the activation process), which may be a substantial improvement for long activation sequences and large cooperativities. The reduction of variability is achieved by having many equivalent multi-step entities, competing to be the first one to activate the whole system. We believe that this mechanism, which we call racing to activation, has not been reported previously in the literature. 
Our focus here is on the general noise suppression mechanism, and not on any specific biological system. Nonetheless, it is clear that various receptor-mediated signaling systems are likely to be examples of where this mechanism will be applicable. The fact that receptors exist on cell surfaces in very large numbers, far in excess of what is needed to fully activate a cell, is encouraging. However, the large number of receptors needed for reduction of the temporal noise in the racing to activation mechanism can come in conflict with the specificity of signaling. It is, therefore, reassuring that, within our model, there is a range of receptor numbers that allows cells to maintain specificity to certain ligands, and yet be activated precisely in time.

Another possible application of our model is in development. There a crucial question is the precision of position determination afforded by morphogen gradients [27, 28, 29]. While most analyses are done for steady state gradients, cells may need to make developmental commitments based on pre-steady state transients [30]. Adding reverse reactions to Fig. 1(a), with rates $k_{\text {reverse }}<k$, our model can be interpreted as a biased (nonequilibrium) random walk of a morphogen particle in the physical space, where the first $m$ of such particles that reach a morphogen detector at $L$ activate it. Our arguments suggest that such temporal, first-passage triggering of developmental commitments can be made very precise.

While too coarse for specific details, our results can be used to make general, verifiable predictions about relations between the number of receptors, the similarity of specific and non-specific ligands, the cooperativity of receptor action on the one hand, and the variability of the activation time on the other. Such data, when available, will shed light on whether the proposed mechanism is used in cellular processes, and to which extent.

\section{Acknowledgments}

IN would like to thank Brian Munsky and Nicolas Hengartner for stimulating discussions during early stages of this work and Byron Goldstein for his help in understanding a proper immune signaling context for our model. The authors are further grateful to attendees of The Sixth q-bio Conference, who provided us with invaluable comments. This work was funded in part by the James S. McDonnell foundation Grant No. 220020321.

\section{References}

[1] D. Coombs, A.M. Kalergis, S.G. Nathenson, C. Wofsy, and B. Goldstein. Activated TCRs remain marked for internalization after dissociation from pMHC. Nat. Immunol., 3:926-931, 2002.

[2] J. Yang and M. Reth. Oligomeric organization of the B-cell antigen receptor on resting cells. Nature, 467:465-469, 2010.

[3] A. Zaidi, M. Saini, and D. MacGlashan Jr. Regulation of Syk kinase and FcRb expression in human basophils during treatment with omalizumab. J. Allergy Clin. Immunol., 125:902-908, 2010. 
[4] D. MacGlashan Jr., B. Bochner, D. Adelman, P. Jardieu, A. Togias, J. McKenzie-White, S. Sterbinsky, R. Hamilton, and L. Lichtenstein. Down-regulation of Fc(epsilon)RI expression on human basophils during in vivo treatment of atopic patients with anti-IgE antibody. $J$. Immunol., 158:1438-1445, 1997.

[5] D. MacGlashan Jr., J. Savage, R. Wood, and S. Saini. Suppression of the basophil response to allergen during treatment with omalizumab is dependent on 2 competing factors. J. Allergy Clin. Immunol., 130:1130-1135, 2012.

[6] C. Fewtrell and H. Metzger. Larger oligomers of IgE are more effective than dimers in stimulating rat basophilic leukemia cells. J. Immunol., 125:701-710, 1980.

[7] C. Torigoe, B. Goldstein, C. Wofsy, and H. Metzger. Shuttling of initiating kinase between discrete aggregates of the high affinity receptor for IgE regulates the cellular response. Proc. Natl. Acad. Sci. USA, 94:1372-1377, 1997.

[8] C. Wofsy, B. Vonakis, H. Metzger, and B. Goldstein. One Lyn molecule is sufficient to initiate phosphorylation of aggregated high-affinity IgE receptors. Proc. Natl. Acad. Sci. USA, 96(15):8615-8620, 1999.

[9] A. Nag, M. Monine, M. Blinov, and B. Goldstein. A detailed mathematical model predicts serial engagement of $\operatorname{IgE}-\mathrm{Fc}($ epsilon)RI complexes can enhance Syk acivation in mast cells. $J$. Immunol., 185:3268-3276, 2010.

[10] O. Dushek, R. Das, and D. Coombs. A role for rebinding in rapid and reliable T cell responses to antigen. PLoS Comput. Biol., 5(11):e1000578, 2009.

[11] S. Valitutti, D. Coombs, and L. Dupre. The space and time frames of T cell activation at the immunological synapse. FEBS Lett., 584(24):4851 - 4857, 2010.

[12] S. Ran and B. Rivnay. Activation of rat basophilic leukemia cells: Temporal identification of the signal calcium influx mediated by the receptor-operated channel pathways. Eur. J. Biochem., 171(3):693-701, 1998.

[13] D. Golan, A.H. Tashjian, E.J. Armstrong, and A. Armstrong. Principles of pharmacology: the pathophysiologic basis of drug therapy. Lippincott Williams \& Wilkins, 2011.

[14] R. P. Stephenson. A modification of receptor theory. Brit. J. Pharmacol., 11(4):379-393, 1956.

[15] M. Nickerson. Receptor occupancy and tissue response. Nature, 178(4535):697-698, 1956.

[16] K. Lee, A. D. Holdorf, M.L. Dustin, A.C. Chan, P.M. Allen, and A.S. Shaw. T cell receptor signaling precedes immunological synapse formation. Science, 295(5559):1539-1542, 2002.

[17] C. DeLisi. The magnitude of signal amplication by ligand-induced receptor clustering. Nature, 289:322-323, 1981.

[18] R. N. Germain. T-cell signaling: The importance of receptor clustering. Curr. Biol., 7(10):R640 - R644, 1997.

[19] J. J. Hopfield. Kinetic proofreading: a new mechanism for reducing errors in biosynthetic processes requiring high specificity. Proc. Nat. Acad. Sci. USA, 71(10):4135-4139, 1974.

[20] J. Ninio. Kinetic amplification of enzyme discrimination. Biochimie., 57(5):587 - 595, 1975.

[21] T.W. McKeithan. Kinetic proofreading in T-cell receptor signal transduction. Proc. Nat. Acad. Sci. USA, 92(11):5042-5046, 1995.

[22] T. Doan, A. Mendez, P. Detwiler, J. Chen, and F. Rieke. Multiple phosphorylation sites confer reproducibility of the rod's single-photon responses. Science, 313:530-533, 2006.

[23] B. Goldstein, D. Coombs, J.R. Faeder, and W.S. Hlavacek. Kinetic proofreading model. Adv. Exp. Med. Biol., 640:82-94, 2008.

[24] B. Munsky, I. Nemenman, and G. Bel. Specificity and completion time distributions of biochemical processes. J. Chem. Phys., 131(23):235103-235103, 2009.

[25] G. Bel, B. Munsky, and I. Nemenman. The simplicity of completion time distributions for common complex biochemical processes. Phys. Biol., 7:0610003, 2010.

[26] F. Rieke and D. Baylor. Single-photon detection by rod cells of the retina. Rev. Mod. Phys., 70:1027-1036, 1998.

[27] F. Tostevin, P.R. ten Wolde, and M. Howard. Fundamental limits to position determination by 
concentration gradients. PLoS Comput. Biol., 3(4):e78, 2007.

[28] T. Gregor, D. Tank, E. Wieschaus, and W. Bialek. Probing the limits to positional information. Cell, 130:153-164, 2007.

[29] A. Lander, W.-C. Lo, Q. Nie, and F. Wan. The measure of success: constraints, objectives, and tradeoffs in morphogen-mediated patterning. Cold Spring Harbor Persp. Biol., 1(1):a002022, 2009.

[30] T Saunders and M Howard. When it pays to rush: interpreting morphogen gradients prior to steady-state. Phys. Biol., 6(4):046020, 2009. 